\section{Forschung für die Energiewende 2.0: integrativ und transformativ}

\author{
von Armin Grunwald und Jens Schippl, ITAS
}

Mit dem Übergang von der Energiewende 1.0 zur Energiewende 2.0 erfolgt ein Perspektivenwechsel von einem eher technischen Fokus hin zu einem Verständnis, das die Energiewende als gesellschaftlichen Transformationsprozess beschreibt. Dementsprechend muss technisch-naturwissenschaftliche Forschung durch sozial- und geisteswissenschaftliche Ansätze ergänzt werden. Vor diesem Hintergrund macht der Beitrag deutlich, dass das zentrale Argument für die Notwendigkeit integrativer Forschung die Aufgabe der Bereitstellung von Handlungswissen ist, welches sich aus unterschiedlichen Wissensbeständen und -kategorien zusammensetzt. Mit dieser Ausrichtung auf außerwissenschaftliche Probleme übernimmt integrative Forschung eine politisch relevante Rolle in der Definition und Bearbeitung von gesellschaftlichen Problemen und wird in ihren Gelingensbedingungen und Qualitätskriterien abhängig vom außerwissenschaftlichen Umfeld. Entscheidend für die Qualität technischer Lösungsangebote ist damit nicht allein die technische Exzellenz, sondern die Passfähigkeit mit gesellschaftlichen Konstellationen.

\section{Traditionelle Energieforschung - notwendig, aber nicht hinreichend}

Die Energiewende in Deutschland verfolgt ambitionierte Ziele. Die Kernkraftwerke sollen bis 2022 abgeschaltet werden und bis 2050 soll der Anteil fossiler Energieträger auf maximal 20 Prozent sinken. Entgegen der verbreiteten Meinung ist das zweite Ziel bei Weitem ambitionierter. Viele Beobachter aus dem Ausland schauen mit zweifelndem Staunen, ob es Deutschland gelingt, den richtigen Kurs einzuschlagen und die Ziele erreichbar zu machen, schließlich gar zu erreichen. In der Tat verlangt die Energiewende an vielen Stellen neue Entwicklungen. Ist das traditionelle Energiesystem bereits technisch und regulatorisch komplex, so wird sich dies durch die
Energiewende noch einmal deutlich verschärfen. Das lässt sich durch ganz unterschiedliche Beispiele belegen: Gerade die stärkere Einbindung von kleineren, dezentralen Produzenten und die Beteiligung von Nutzern am Management des Gesamtsystems erfordern neue Regelungen und Anreizstrukturen, aber auch neue Wege der Datenübertragung, -interpretation und -vorhaltung, die jeweils die Komplexität des Gesamtsystems erhöhen (Büscher/Schippl in diesem Heft). $\mathrm{Zu}$ den nicht-technischen Handlungsfeldern gehört die Entwicklung von Anreizsystemen zur Einrichtung und Integration von systemstabilisierenden Elementen wie Speichern. Ebenso gehören dazu die Entwicklung neuer Geschäftsmodelle und Dienstleistungen im Energiebereich, die Weiterentwicklung von Planungsrecht und Beteiligungsverfahren, um sozialverträglich neue Infrastrukturen einrichten zu können (Schweizer/Renn in diesem Heft), die Koordinierung auf unterschiedlichen Ebenen der Governance (Ohlhorst et al. in diesem Heft) und die Ausarbeitung von vielversprechenden Innovationspfaden. Die zukünftige Entwicklung des Systems wird somit stark von Wechselwirkungen zwischen technischen und nicht-technischen Faktoren geprägt sein. Entsprechend wird der Erfolg der Transformation davon abhängen, wie gut das Zusammenwirken beider Bereiche funktioniert.

Ist zwar das Energiesystem ,immer schon“ ein soziotechnisches System (Grunwald 2012), so wächst die Bedeutung des „Sozio“-Anteils durch die Energiewende. Zunehmend gerät dies in das gesellschaftliche Bewusstsein. War die Energiewende anfänglich von einer „grünen“ Technikgläubigkeit geprägt, welche darin v. a. den Ersatz alter (nuklearer und fossiler) durch neue (erneuerbare und effiziente) Technologien sah, dämmert es nun allmählich, dass dieser Blick verkürzt, wenn nicht naiv ist. Exzellente Technik ist eine zwar sicherlich notwendige, aber nicht hinreichende Bedingung für einen gelingenden Transformationsprozess. Bestes Indiz ist die politisch und massenmedial geführte Debatte. Denn in ihr geht es nicht um technische Herausforderungen und Probleme, sondern um einen gerechten Strompreis, um die Ablehnung von Pumpspeicherkraftwerken durch die lokale Bevölkerung und um Sorgen vor elektromagnetischer Strahlung durch neue Hochspan- 
nungstrassen (Stromautobahnen). Das sind sämtlich keine technischen, sondern soziale Herausforderungen. Die Energiewende stellt sich damit als doppelte Herausforderung dar: technisch wie gesellschaftlich (Schippl/Grunwald 2012). Wie bereits in der Einführung zum Schwerpunkt in diesem Heft dargelegt, symbolisiert das Wort von der Energiewende 2.0 den Übergang von einer naiven Vorstellung, die Energiewende (1.0) sei v. a. der Ersatz alter durch neue Technologien hin zu einem Verständnis, dass die Energiewende als tief in die Gesellschaft eingreifende Transformation begreift.

Dementsprechend erschöpfen sich die Möglichkeiten der Wissenschaft, zur Transformation beizutragen, keineswegs im Bereitstellen neuer Technologien und in der wirtschaftswissenschaftlichen Erforschung von neuen Geschäftsmodellen und Dienstleistungen. Sozial- und Geisteswissenschaften sind gefragt, sobald es um die gesellschaftlichen, politischen, kulturellen, psychologischen und ethischen Fragen geht. Denn ein erfolgreiches Design der benötigten Innovationen und Prozesse für den Umbau des Energiesystems und eine entsprechende Gestaltung der Schnittstellen zu Nutzern und Bürgern setzt auch solches Wissen voraus. Während die Energieforschung bisher vielfach auf die Entwicklung neuer Technologien fokussiert ist, geht es nun darum, auch mögliche oder notwendige Veränderungen auf der „,Sozio“Seite des Energiesystems in den Mittelpunkt des Interesses zu stellen. Hierzu müssen Wissensbestände unterschiedlicher Disziplinen zusammen gebracht werden, interdisziplinäre und integrative Forschung ist erforderlich. Nicht nur innovative Technologien, sondern auch neue Ansätze der Planung, Steuerung, Anpassung, Kommunikation und Partizipation werden benötigt.

Forschung für die Energiewende muss zwar die klassische, durch Technikentwicklung einerseits und Energiewirtschaft andererseits geprägte Energieforschung notwendigerweise enthalten. Jedoch muss in doppelter Hinsicht etwas hinzutreten: Zum einen müssen die hier nur angedeuteten sozialen Aspekte der Energiewende durch sozialwissenschaftliche Forschung in den Blick genommen werden. Konsum- und Verhaltensforschung, Governance-Forschung und politische Wissenschaft, Planungsverfahren und ethische Reflexionen der Kriterien für Entscheidungs- findungen gehören eben auch zur Energieforschung. ${ }^{1}$ Zum zweiten dürfen diese Stränge der Energieforschung, der technikwissenschaftliche, der wirtschaftswissenschaftliche und der sozialwissenschaftliche Strang nicht unverbunden nebeneinanderher betrieben werden, sondern müssen ihre Fragen und Ergebnisse jeweils im Kontext der anderen diskutieren, damit die Bedingung der Möglichkeit der Erzeugung konsistenter Gesamtbetrachtungen überhaupt gegeben ist. Aus diesem Grund wird im vorliegenden Beitrag von der Notwendigkeit einer integrativen Energieforschung gesprochen (Kap. 2). Ob und in welcher Hinsicht diese auch transformativ sein muss, bedarf einer eigenen Überlegung (Kap. 3). Der Beitrag schließt mit einer Reihe zusammenfassender Thesen, welche die Bedeutung integrativer Forschung für die Energiewende 2.0 herausstreichen.

\section{Charakteristika integrativer Energiefor- schung}

Der Integrationsbegriff wird in Bezug auf Forschung durchaus in verschiedener Weise verwendet. Geht es in interdisziplinärer Hinsicht v. a. um die Integration von Wissensbeständen verschiedener Herkunft (Grunwald 2001), so bezieht sich die Integration ökonomischer Sektoren eher auf eine Verbreiterung des Gegenstandsbereiches der Forschung zur Energiewende, und die Integration der Dimensionen der Nachhaltigkeit auf die Einordnung in ein kohärentes Zielsystem (Kopfmüller et al. 2001). Entscheidend dafür, was jeweils im Vordergrund steht und was genau integriert werden soll, ist die Zielrichtung und die „Mission“ der betreffenden Forschung.

Ohne eine wissenschaftsexterne Aufgabenstellung würde kaum integrative Forschung betrieben. Der Grund für das integrative Herangehen der Forschung an Problemanalysen und an Beiträge zu Problemlösungen in der Energiewende liegt keineswegs einfach in der Komplexität der Untersuchungsgegenstände oder Forschungsfragen. Komplexe Untersuchungsgegenstände können auch monodisziplinär und sektoral bearbeitet werden, wenn nämlich die einzelnen Disziplinen im Lichte ihres jeweiligen Erkenntnisinteresses, ihrer Methoden, Begriffsbildungen und Forschungskonzeptionen heran- 
gehen. Zentrales Argument für die Notwendigkeit integrativer Forschung ist nicht einfach die Komplexität, sondern letztlich die Aufgabe der Bereitstellung von Handlungswissen. ${ }^{2}$

Das Integrationspostulat folgt aus der Notwendigkeit, zu integriertem und kohärentem politischen, wirtschaftlichen und gesellschaftlichen Handeln zur Zukunft der Energieversorgung zu kommen. Die Integration disziplinären und sektoralen, evtl. auch außerwissenschaftlichen Wissens (dann wäre integrative Forschung auch transdisziplinär) erfolgt unter Problemlösedruck (Bechmann/Frederichs 1996). Diesem kann nur pragmatisch (zielend auf den Bereitstellung und Beurteilung von Handlungs- und Entscheidungswissen, von Instrumenten und Strategien sowie im Hinblick auf Umsetzung), kontextbezogen und eingebettet in gesellschaftliche und politische Konstellationen Rechnung getragen werden.

Das von integrativer Forschung für die Zwecke der Politikberatung, der demokratischen Debatte, ökonomischer Entscheidungen und auch für die weitere Ausgestaltung der wissenschaftlichtechnischen Agenda bereitgestellte „Wissen zum Handeln" soll über Beiträge zur wissenschaftlichen Erkenntnis hinaus einen „Impact“ in der außerwissenschaftlichen Welt erzielen (Decker/ Ladikas 2004). Es unterliegt damit zweierlei Qualitätskriterien: zum einen den üblichen Kriterien wissenschaftlicher Validität und Qualität, zum anderen aber auch Kriterien der Adäquatheit an gesellschaftliche Problemlagen und der Eignung zur Problemlösung (Grunwald 2001). Dieses Wissen besteht aus unterschiedlichen Anteilen:

- Normative Orientierung: Die Beurteilung von Zielen der Energiewende, Maßnahmen zur Umsetzung einschließlich neuer Technologien und ihrer Folgen bedarf einer normativen Orientierung, von rechtlichen bis hin zu ethischen Überlegungen, z. B. zur Wünschbarkeit von Zielen und der Akzeptabilität von Risiken (dieser Wissenstypus wird gelegentlich auch als Zielwissen bezeichnet; vgl. Schneidewind/Singer-Brodowski 2013).

- Systemwissen: Ein hinreichendes Verständnis des Energiesystems (technische Entwicklungen und ihre Bedingungen, Anwendungsmöglichkeiten, Akteurskonstellationen und Interessen, Erfolgsbedingungen und hemmende
Faktoren für Innovationen, Wechselwirkungen zwischen Wissenschaft, Technik, Politik, Umwelt und Gesellschaft etc.) ist notwendige Voraussetzung zur Bereitstellung von integrativem Wissen. Dieser Wissenstypus kann häufig durch disziplinäre (auch sozialwissenschaftliche) Forschung bereitgestellt und in integrative Prozesse eingebracht werden.

- Zukunftswissen: Entwicklung und Erforschung von Energiezukünften sind als spezifische Orientierungen zu möglichen zukünftigen Entwicklungen im Energiebereich erforderlich, um unterschiedliche Wege in die Zukunft hinein zu strukturieren und sie für Entscheidungssituationen fruchtbar $\mathrm{zu}$ machen. Diese Formen prospektiven Wissens sind gelegentlich Prognosen, häufiger aber Erschließungen zukünftiger Entwicklungen mit niedrigerem Geltungsanspruch, wie v. a. Energieszenarien (Dieckhoff et al. 2011).

- Handlungswissen: Wissen über Wirkungen von Maßnahmen, z. B. der Regulierung oder der Innovationsförderung, ihre Zielerreichung und Effizienz genauso betreffend wie mögliche unbeabsichtigte Nebenfolgen, stellt eine entscheidende Voraussetzung einer informierten und rationalen Entscheidungsfindung in der Energiepolitik dar. Dieses Wissen muss aus einer Vielzahl von Wissensbeständen aggregiert werden, darunter auch Elemente aus den vorhergehend genannten Wissenskategorien.

Die Integration, teils auch bereits die Bereitstellung dieser Wissenselemente bedürfen eines zumindest inter-, bei der Integration außerwissenschaftlicher Wissensbestände und Forschungspartner auch transdiziplinären Zugangs. Politikwissenschaftliche, betriebs- und volkswirtschaftliche, umweltbezogene, soziale, kulturelle, technische, sozial- oder individualpsychologische und ethische Aspekte müssen, ohne Anspruch auf Vollständigkeit, interdisziplinär integriert und ggf. transdisziplinär um das außerwissenschaftliche „lokale Wissen“von Betroffenen und ihre Beurteilungsperspektiven ergänzt werden. Entsprechend breit ist das zum Einsatz kommende Methodenspektrum (Grunwald 2010, Kap. 7).

In gewisser Weise tritt dadurch Wissenschaft nicht rein erkenntnisorientiert, sondern als „Notwissenschaft“ auf (Lorenzen 1987). Das 
Erkenntnisinteresse problemorientierter Forschung (Bechmann/Frederichs 1996) ist nicht (nur) durch Natur- oder Welterkenntnis geprägt, wie dies in deren Selbstinterpretation in den klassischen Disziplinen dominant ist, sondern durch den Problem- und Entscheidungsbezug des kreierten Wissens. Integrative Energieforschung begibt sich aus der geschützten Nische der vermeintlichen Wertfreiheit heraus, übernimmt eine politisch relevante Rolle in der Definition und Bearbeitung von gesellschaftlichen Problemen und wird in ihren Gelingensbedingungen und Qualitätskriterien abhängig vom außerwissenschaftlichen Umfeld (Grunwald 2001).

\section{Energieforschung - aktive Intervention statt distanzierter Beobachtung?}

Wie in Kapitel 1 skizziert, ist die Energiewende mit einer Neuformierung vieler gesellschaftlicher Handlungsfelder verbunden - und das bedeutet, dass die Energiewende nicht einfach die Substitution alter durch neue Technik, sondern ein Stück weit Selbsttransformation der Gesellschaft ist. Diese Erkenntnis hat weitreichende Folgen, v. a. weil über die Neuformierung gesellschaftlicher Handlungsroutinen im Kontext soziotechnischer Transformationen ein prognostisches Wissen in vielen wichtigen Bereichen kaum verfügbar ist. Diese Tatsache wird zu selten direkt angesprochen, dabei lassen sich einschlägige Beispiele ohne Weiteres den aktuellen Diskussionen zur Energiewende entnehmen: Ob, auf welche Weise und in welchem Umfang ein „Demand Side Management" zur Systemstabilität praktisch beitragen wird; wie sich die Nutzer in einem Smart Grid verhalten werden, wenn sie sich einmal über Jahre hinweg an den Umgang mit der Technologie gewöhnt haben (oder vielleicht als ,SmartGrid-Natives" sogar damit aufgewachsen sind); welche Rolle der zwischenstaatliche europäische Ausgleich spielen wird; wie rasch Effizienzgewinne und Stromeinsparung möglich werden; ob sich die Kosten für Photovoltaik-Anlagen weiterhin ,überraschend“ stark reduzieren; ob Elektromobilität wirklich der Königsweg ist, den Abschied von fossilen Energieträgern im Mobilitätsbereich einzuläuten; wie stark das zukünftige Energiesystem dezentralisiert sein wird; wie sich politische Rahmenbedingungen und ökonomische Anreizsysteme entwickeln werden - all das ist heute nicht wissbar, sondern muss sich erst herausstellen. Damit kann die Energiewende nicht im Modus klassischer Planung betrieben werden, sondern muss mit großen Unsicherheiten und Offenheiten umgehen. Die jeweils nächsten Schritte müssen trotz der Langfristanforderungen der Energiewende inkrementell vorgenommen werden -allerdings nicht in beliebiger, sondern durch die langfristigen Zielvorgaben und das Leitbild der Nachhaltigkeit orientierter Weise. Dabei entstehen neue Konflikte, insbesondere zwischen dem Wunsch nach Flexibilität und Reflexivität einerseits (Voß et al. 2006) und den Anforderungen an Planungssicherheit und Festlegung. Eine der zentralen Herausforderungen der Energiewende ist es hier einerseits, eine zumindest mittelfristige verlässliche und gegen politische Wechsel einigermaßen robuste Investitionssicherheit zu geben; anderseits aber auch die Möglichkeit offen zu lassen, um auf die, zu einem Lernprozess dazu gehörenden Fehler zeitnah und angemessen zu reagieren (Schippl 2013).

Damit verschärfen sich die Herausforderungen an die Beiträge der Wissenschaft. Integrative Energieforschung muss inmitten von laufenden Transformationsprozessen möglichst „robustes“ Wissen für demokratische Debatten und politische Entscheidungsprozesse bereitstellen. Integrative Energieforschung ist einerseits Teil eines umfassenden Transformationsprozesses und gestaltet diesen andererseits, und zwar gleichzeitig, durch Bereitstellung von Wissen und Orientierung „,im Prozess“" mit. Allgemeiner ist sie nur als Bestandteil von transformativen Prozessen denkbar, in denen sich erst Schritt für Schritt der Weg in eine nachhaltigere Energiezukunft erschließt (Grunwald 2007). Strategisches Wissen, bereitgestellt durch integrative Energieforschung, ist auf diese Weise Teil eines umfassenderen transformativen Wissens, bestehend aus System-, Zukunfts-, Orientierungs- und Handlungswissen:

„Transdisziplinäre Forschung ist nicht mehr ausschließlich Wissensproduzent, sondern zunehmend auch Wissensanwalt und ,Wissensbroker`. Transdisziplinäre Forschung katalysiert Veränderungsprozesse in der Gesellschaft, damit wird sie zur ,transformativen Wissenschaft‘." (Schneidewind/Singer-Brodowski 2013) 
Es steht außer Frage, dass sich solche Veränderungsprozesse nicht an den Grenzen wissenschaftlicher Disziplinen orientieren oder gar mit einer einzigen Disziplin erfasst werden können. Transformative Forschung muss also mindestens interdisziplinär sein. Einzelne Disziplinen können dafür durchaus Wissen bereitstellen, welches häufig Systemwissen im obigen Sinne sein wird. Eine explizit transformative Wissenschaft muss sich aber problemorientiert aus dem Portfolio unterschiedlicher Wissensbestände und Wissenstypen bedienen und diese ohne Rücksicht auf disziplinäre Grenzen integrieren. Hier sind zwei Stufen der Integration zu unterscheiden: (a) die Integration von wissenschaftlichen Wissensbeständen und (b) die Einbettung des integrativen Wissens in praktische Kontexte, in denen z. B. über neue EnergieInfrastrukturen entschieden wird. Das erste ist dabei die Voraussetzung für das zweite.

Die in der Einleitung und in vielen Beiträgen dieses Schwerpunktes vorgestellte Helmholtz-Allianz ENERGY-TRANS zeigt, wie interdisziplinäre Energieforschung aufgestellt sein kann, die das Wechselspiel zwischen technischen Potenzialen, Innovationsprozessen, Nutzerverhalten, politischen und ökonomischen Randbedingungen, Konflikten sowie Steuerungsprozessen in den Blick nimmt (vgl. Schippl et al. 2012; http://www.energy-trans.de). Dabei hat sich die Allianz primär dem ersten der beiden Schritte verschrieben. Diese Forschung ist sicher integrativ in dem oben ausgeführten Sinn und orientiert sich an außerwissenschaftlichen Problemdiagnosen. Die Allianz hat sich insgesamt sowie auch in den einzelnen Teilbereichen eine wissenschaftsexterne Aufgabenstellung gegeben. Damit verbunden hat sie sich ins Programm geschrieben, nicht nur wissenschaftlich verwertbares Wissen, sondern eben auch ,knowledge for action" bereitzustellen. Damit ist die Allianz jedoch nicht automatisch auch transformativ.

Letztlich muss sich der Erfolg der Allianz aber daran messen lassen, ob auch transformatives Wissen entstanden ist, also Wissen, dass im Rahmen von Transformationsprozessen nicht nur verwendet werden kann, sondern auch verwendet wird. Um dies sicherzustellen, wird in der weiteren Arbeit in der Allianz bzw. in Erweiterungs- oder Folgevorhaben die bisherige interdisziplinäre Ausrichtung zu einer transdisziplinären erweitert werden müssen. Dazu muss die Zusammenarbeit mit Praxispartnern auf verschiedenen Ebenen etabliert werden, was z. B. im Rahmen von „Reallaboren“ (WfN 2013) erfolgen kann: Transdisziplinäre Forschung benötigt Orte, an denen gesellschaftliche Transformationsherausforderungen mit transdisziplinären Forschungs- und Lehrdesigns verknüpft werden. Reallabore bieten hierfür ideale Voraussetzungen. Dabei handelt es sich um reale gesellschaftliche Kontexte (Städte-/Stadtteile, Regionen, Branchen, komplexe Stoffkreisläufe), bei denen eine wissenschaftsgeleitete Unterstützung des Transformationsprozesses im Sinne eines transdisziplinären Prozesses erfolgt (WfN 2013).

\section{Zusammenfassende Thesen}

Energieforschung für die Energiewende 2.0 ist nicht nur für einzelne Disziplinen, sondern für Wissenschaft insgesamt eine Herausforderung - v. a., weil traditionelle Schemata der Wissenschaften wie Disziplinen, Unabhängigkeit und das Selbstverständnis als distanzierte Beobachter zwar nicht obsolet werden, aber herausgefordert werden und teils neu gedacht werden müssen. Der deutsche Wissenschaftsrat hat dies so ernst genommen, dass er eine Arbeitsgruppe eingerichtet hat, welche sich mit den strukturellen Herausforderungen der „challenge-getriebenen“ Wissenschaft anlässlich der Energiewende befasst. In diesem Kontext lassen sich vor dem Hintergrund der oben vorgetragenen Überlegungen folgende zusammenfassende Thesen formulieren.

1. Energieforschung muss eine erhebliche thematische Diversität bearbeiten, da die Energiewende eine gesellschaftliche Selbsttransformation und nicht nur der Ersatz alter durch neue Technik ist. Die Vielfalt reicht von den Technik- und Naturwissenschaften über Wirtschafts- und Sozialwissenschaften bis hin zu Rechts- und Kulturwissenschaften sowie Philosophie.

2. Technische Forschung und Entwicklung verliert nichts von ihrer Bedeutung. Sie muss jedoch zusehends darauf achten, nicht zugunsten rein technischer Optimierung zu arbeiten, 
sondern muss die Forschung an einem erheblich breiter angelegten Satz soziotechnischer Kriterien ausrichten.

3. Entscheidend für die Qualität technischer Lösungsangebote ist damit nicht allein die technische Exzellenz, sondern die Passfähigkeit mit gesellschaftlichen Konstellationen. Es geht nicht um exzellente Technik als solche, sondern um funktionierende und im Sinne der Energiewende zielführende soziotechnische Wechselwirkungen.

4. Analoges gilt für wirtschafts- und sozialwissenschaftliche Forschung. Auch sie muss einerseits disziplinäre Fragen im Kontext der Energiewende identifizieren und nach disziplinären Standards bearbeiten - andererseits aber in der Lage sein, diese Forschung in einen integrativen Kontext einzubetten, und zwar sowohl in Bezug auf die bearbeiteten Themen als auch im Hinblick auf die Ergebnisse.

5. Sozialwissenschaftliche Forschung darf nicht auf die Bereitstellung sozialtechnologischen Rezeptwissens, also z. B. wie man Akzeptanz generieren soll, reduziert werden. Dieses würde die legitimen Mitwirkungs- und Beteiligungsinteressen von Stakeholdern, Zivilgesellschaft und BürgerInnen ignorieren. Versuche der direkten Akzeptanzbeschaffung für bereits getroffene Entscheidungen sind in einer offenen Gesellschaft zum Scheitern verurteilt.

6. Distanzierte Beobachtung gesellschaftlicher Entwicklungen bleibt notwendiger Bestandteil sozialwissenschaftlicher Forschung. Jedoch verstärkt sich die Notwendigkeit, auch experimentell und gestaltend einzugreifen und damit zu einem ,transformativen Wissen“ beizutragen (s. o.). Dies kann z. B. in Form von „Reallaboren“" (s. o.; WfN 2013) erfolgen.

7. Diese Form wissenschaftlichen Engagements erhöht die Anforderungen an Transparenz in normativen und epistemologischen Fragen beträchtlich, damit in den Wissenschaften Eigenständigkeit und die Möglichkeit zur Kritik bewahrt bleiben und sie nicht zu einem Stakeholder neben anderen werden.

8. Die Entwicklung und Anwendung transformativen Wissens will gelernt sein. Transformative Forschung und Projekte wie ENERGY-TRANS brauchen ihren Platz im Wissen- schaftssystem, sowohl in Forschung als auch in Lehre. Damit ist auch die Forderung nach hinreichender Stabilität und Kontinuität verbunden, um Methodenentwicklung betreiben und geeignete Formen der Tradierung entwickeln zu können.

9. Auch der Umgang mit Nicht-Wissen will gelernt sein. So haben die Beiträge in diesem Schwerunkt auch verdeutlicht, dass Unsicherheit und Nicht-Wissen im Kontext der Energiewende hinsichtlich vieler erfolgsentscheidender Parameter nach wie vor hoch sind. In vielen Bereichen fehlt es an Zielwissen, Systemwissen und dementsprechend auch an Zukunftswissen und Handlungswissen. Experimentieren darf hier nicht mit Planlosigkeit verwechselt werden. Genauso wie die Energiewende nicht ohne Kosten zu haben ist, wird sie auch nicht ohne Fehler zu haben sein. Nachträgliches Korrigieren von Entscheidungen ist angesichts der Komplexität des Energiesystems unumgänglich und zweifellos erfolgsentscheidend.

\section{Anmerkungen}

1) Sehr früh, d. h. schon vor der Energiewende, hat auf diese Anforderungen an eine moderne Energieforschung die Berlin-Brandenburgische Akademie der Wissenschaften hingewiesen (Leopoldina, acatech, BBAW 2009).

2) In einem extrem weiten Verständnis von Transdisziplinarität ist bereits Forschung, die sich an wissenschaftsexternen Zielen orientiert, transdisziplinär (Mittelstraß 1998). Damit wäre integrative Forschung per definitionem auch transdisziplinär. Diesem Verständnis folgen wir jedoch nicht. Es ist für unsere Zwecke zu allgemein, da z. B. auf diese Weise bereits jede Industriekooperation der Ingenieurwissenschaften transdisziplinär wäre. Wir gehen vielmehr von einem Verständnis von Transdisziplinarität aus, das die Kooperation mit außerwissenschaftlichen Akteuren im Forschungsprozess als zentrales Kriterium ansieht (Schneidewind/Singer-Brodowski 2013; WfN 2013).

\section{Literatur}

Bechmann, G.; Frederichs G., 1996: Problemorientierte Forschung: Zwischen Politik und Wissenschaft. In: Bechmann, G. (Hg.): Praxisfelder der Technik- 
folgenforschung. Konzepte, Methoden, Optionen. Frankfurt a. M., S. 11-37

Decker, M.; Ladikas, M. (Hg.), 2004: Bridges Between Science, Society and Policy. Technology Assessment - Methods and Impacts. Berlin

Dieckhoff, Chr.; Fichtner, W.; Grunwald, A. et al. (Hg.), 2011: Energieszenarien. Konstruktion, Bewertung und Wirkung - „Anbieter“ und „Nachfrager“ im Dialog. Karlsruhe

Grunwald, A., 2001: Integrative Forschung zum Globalen Wandel - Herausforderungen und Probleme. In: Coenen, R. (Hg.): Integrative Forschung zum globalen Wandel. Frankfurt a. M., S. 23-48

Grunwald, A., 2007: Working Towards Sustainable Development in the Face of Uncertainty and Incomplete Knowledge. In: Journal of Environmental Policy \& Planning 9/3 (2007), S. 245-262

Grunwald, A., 2010: Technikfolgenabschätzung. Eine Einführung. Berlin

Grunwald, A., 2012: Technikzukünfte als Medium gesellschaftlicher Zukunftsdebatten und der Technikgestaltung. Karlsruhe

Kopfmüller, J.; Brandl, V.; Jörissen, J. et al., 2001: Nachhaltige Entwicklung integrativ betrachtet. Konstitutive Elemente, Regeln, Indikatoren. Berlin

Leopoldina - Deutsche Akademie der Naturforscher/ Nationale Akademie der Wissenschaften; acatech Deutsche Akademie der Technikwissenschaften; BBAW - Berlin-Brandenburgische Akademie der Wissenschaften, 2009: Konzept für ein integriertes Energieforschungsprogramm für Deutschland. Berlin; http:// www.acatech.de/fileadmin/user_upload/Baumstruktur_nach_Website/Acatech/root/de/Aktuelles__Presse/Presseinfos__News/Energiepapier_final_1_.pdf (download 23.7.13)

Lorenzen, P., 1987: Lehrbuch der konstruktiven Wissenschaftstheorie. Mannheim

Mittelstraß, J., 1998: Interdisziplinarität oder Transdisziplinarität? In: Mittelstraß, J. (Hg.): Die Häuser des Wissens. Frankfurt a. M., S. 29-48

Schippl, J., 2013: Die Energiewende als gesellschaftliche Herausforderungen: Interdisziplinäre Forschung in der Helmholtz-Allianz ENERGY-TRANS. Vortrag beim 17. Fachkongress Zukunftsenergien im Rahmen der E-world energy \& water 2013. Essen, 5.2.13

Schippl, J.; Grunwald, A., 2012: Energieinnovationen zwischen Gesellschaft und Technik: Die HGF-Allianz ENERGY-TRANS. Vortrag auf dem Symposium „Energieinnovation“. Graz, Österreich, 15.-17.2.12

Schippl, J.; Grunwald, A.; Renn, O., 2012: Zukünftige Infrastrukturen der Energieversorgung. Helmholtz-
Allianz ENERGY-TRANS gegründet. In: GAIA 21/3 (2012), S. 242-243

Schneidewind, U.; Singer-Brodowski, M., 2013: Transformative Wissenschaft. Klimawandel im deutschen Wissenschafts- und Hochschulsystem. Marburg Voß, J.-P; Bauknecht, D.; Kemp, R. (Hg.), 2006: Reflexive Governance for Sustainable Development. Cheltenham

WfN - Wissenschaft für Nachhaltigkeit. Expertengruppe, 2013: Ministerium für Wissenschaft und Kunst. Stuttgart

\section{Kontakt}

Prof. Dr. Armin Grunwald Institut für Technikfolgenabschätzung und Systemanalyse (ITAS)

Karlsruher Institut für Technologie (KIT)

Karlstraße 11, 76133 Karlsruhe

Tel.: +49 721 608-22500

E-Mail: armin.grunwald@kit.edu 